\title{
Development of Lichen Planopilaris-Like Alopecia following Occupational Exposure to Trichloroethylene and Tetrachloroethylene
}

\author{
Dustin H. Marks ${ }^{a}$ Dina Hagigeorges ${ }^{a}$ Athena J. Manatis-Lornella \\ Ruth K. Foreman ${ }^{b, c}$ Maryanne M. Senna ${ }^{a, c}$ \\ ${ }^{a}$ Department of Dermatology, Massachusetts General Hospital, Boston, MA, USA; ${ }^{b}$ Dermatopathology Service, \\ Massachusetts General Hospital, Boston, MA, USA; ${ }^{C}$ Harvard Medical School, Boston, MA, USA
}

\section{Established Facts}

- Lichen planopilaris (LPP) and its variant form frontal fibrosing alopecia (FFA) represent primary lymphocytic cicatricial alopecias.

- While the exact pathogenesis is unknown, it is suspected that genetic, hormonal, and environmental factors may contribute to the development and severity of LPP and FFA.

\section{Novel Insights}

- Acute exposure to trichloroethylene and tetrachloroethylene may be associated with development of LPP-like alopecia.

\section{Keywords}

Cicatricial alopecia $\cdot$ Scarring alopecia $\cdot$ Lichen planopilaris - Chemical exposure - Trichloroethylene .

Tetrachloroethylene · Perchloroethylene · Gorlin syndrome

\begin{abstract}
Background: We report a case of acute and severe lichen planopilaris (LPP)-like alopecia in a 35-year-old male construction worker following occupational exposure to trichloroethylene (TCE) and tetrachloroethylene (PCE). Case $\boldsymbol{R} \boldsymbol{e}$ port: Two weeks after initiating ground-intrusive construction at a previous dry-cleaning facility site, the patient
\end{abstract}

(c) 2019 S. Karger AG, Basel

E-Mail karger@karger.com www.karger.com/sad developed sudden scalp pruritus and associated patchy hair loss. As subsequent scalp biopsies revealed LPP, he was started on hydroxychloroquine at $200 \mathrm{mg}$ twice daily and clobetasol solution once daily. Despite treatment, the patient's hair loss rapidly progressed to involve $>95 \%$ of his scalp within 3 years. An official "work clean" policy report revealed high-concentration exposure to TCE and PCE. Conclusion: Although causation cannot be proven, the close temporal relationship and rapid progression of LPP-like alopecia in an atypical patient demographic support a strong correlation between chemical exposure to TCE/PCE and scarring hair loss.

(c) 2019 S. Karger AG, Basel 


\section{Introduction}

Lichen planopilaris (LPP) and its variant form frontal fibrosing alopecia (FFA) represent lymphocytic cicatricial alopecias involving destruction of the stem cells located in the bulge region of the hair follicular unit [1]. While remaining rare forms of hair loss, the incidence of LPP and FFA has significantly increased over the last two decades since the first report of FFA in 1994 [2, 3]. Given the recent emergence, it is postulated that an environmental influence could contribute to the pathogenesis, in addition to suspected genetic and hormonal factors [3].

Moreover, a genome-wide association study identified four genomic loci associated with FFA, including a putative causal missense variant that encodes for a homonymous xenobiotic-processing enzyme [4]. In an attempt to identify potentially relevant exposures, the existing literature has focused on the association of FFA with leave-on facial skin care products and sunscreen, but no other significant findings have been reported to date [5]. Herein, we describe the acute development of LPP-like alopecia in a male construction worker exposed to trichloroethylene (TCE) and tetrachloroethylene (PCE).

\section{Case Report}

A 35-year-old Caucasian male with a history of Gorlin syndrome presented with scalp and body hair loss. He had had no dermatologic history until 6 months prior to presentation, when he was digging an underground tunnel at a previous dry-cleaning facility site for several months. He wore the mandated personal protective equipment including a hard hat and hazmat suit from the neck down, leaving his face and scalp largely exposed. Two weeks after starting construction, the patient experienced new onset of severe scalp pruritus with sudden, substantial hair loss on the scalp and upper and lower extremities. The patient was seen by an outside dermatologist, who performed a punch biopsy on the right thigh, which was most consistent with a primarily lymphocytic cicatricial alopecia, favoring LPP. Topical steroids and tacrolimus, intralesional triamcinolone, and an oral prednisone taper were tried without benefit, and the patient subsequently sought a second opinion.

Physical examination demonstrated a $4-\mathrm{cm}$ patch of scarring hair loss and moderate perifollicular erythema and scale on the left frontal crown scalp in addition to subtle areas of scarring hair loss on the central vertex (Fig. 1). In addition, there were numerous folliculocentric erythematous papules and pustules with associated hair loss (Fig. 2) on the bilateral arms, waistline, and buttocks. A laboratory workup with a complete blood count and comprehensive metabolic panel showed results within normal limits, and repeat biopsies of the left crown and vertex scalp were again consistent with a primarily lymphocytic cicatricial alopecia (Fig. 3, 4). The patient was started on $200 \mathrm{mg}$ hydroxychloroquine twice daily and clobetasol solution once daily for his hair loss, and clindamycin lotion twice daily for the acneiform lesions.

Development of LPP following Exposure to TCE/PCE

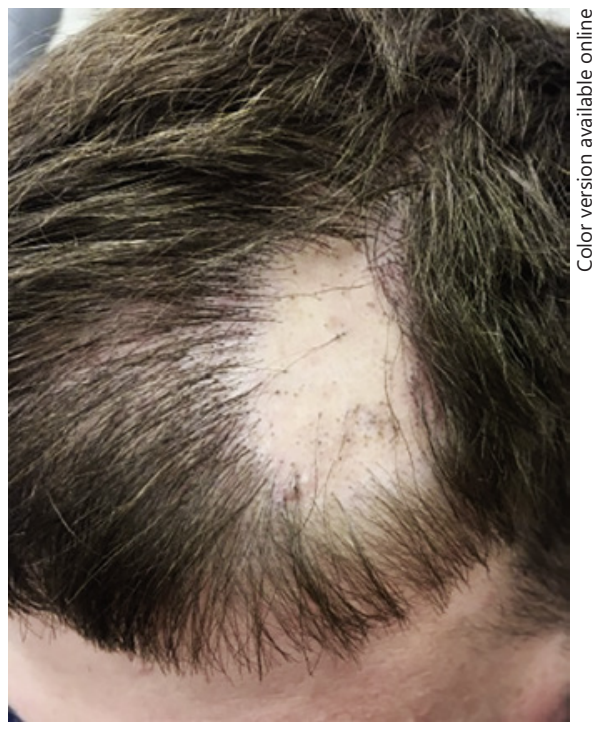

Fig. 1. Scalp at initial visit. A 4-cm patch of scarring hair loss with follicular plugging and perifollicular erythema and scale along the perimeter on the left frontal crown scalp can be seen.

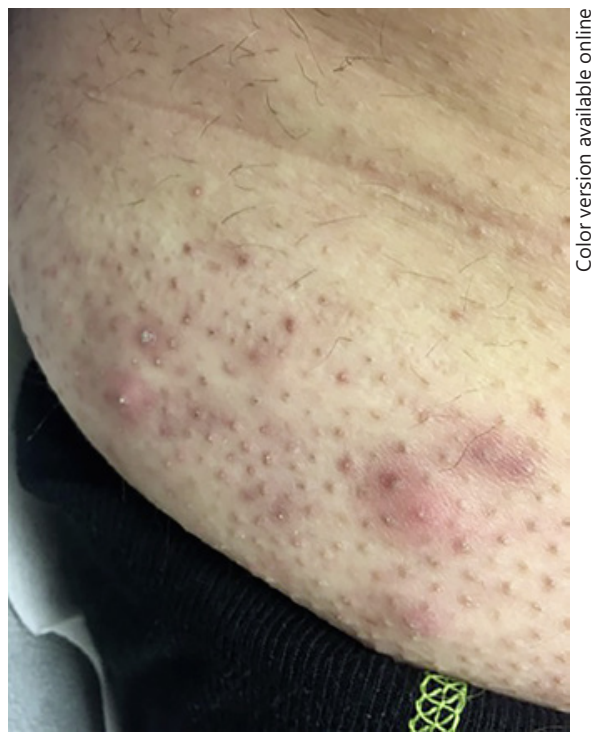

Fig. 2. Waistline at initial visit. Right waistline with numerous folliculocentric erythematous papules and pustules.

At the 1-month follow-up, the patient demonstrated significant worsening of the open and closed comedones, pustules, and cysts with thick acantholytic hair casts most prominent at the waistline and gluteal folds. Suspicion for chloracne was raised, and bacterial cultures from a prominent pustule grew normal flora. The patient denied any improvement with a 7-day course of $500 \mathrm{mg}$ cephalexin 3 times daily but eventually experienced resolution with tazarotene $0.1 \%$ cream. Due to his suspected chloracne and unusual demographic for LPP, we were concerned for a toxic 


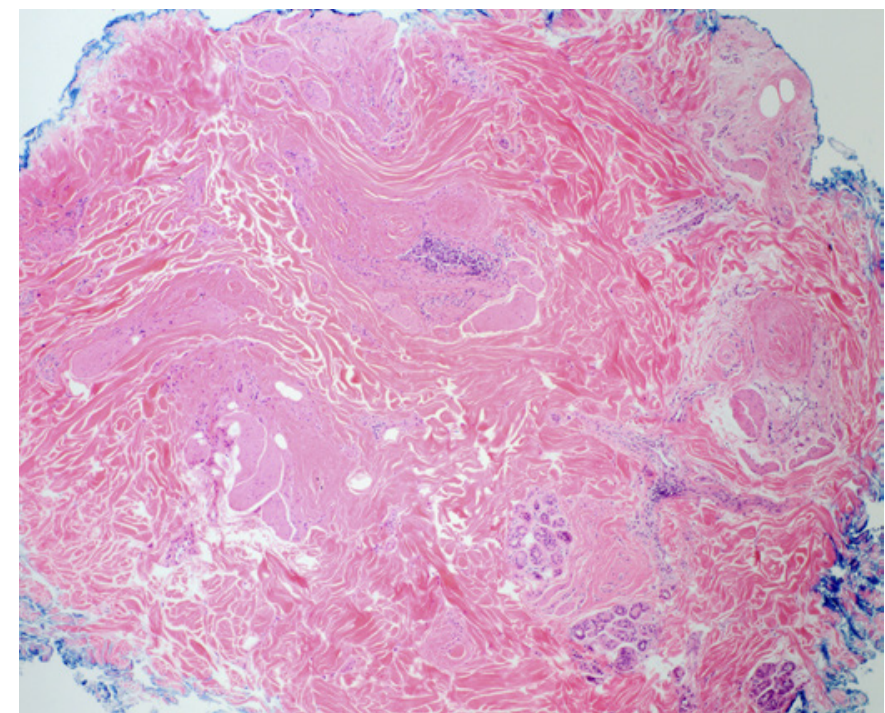

Fig. 3. H\&E stain. Low-power image. Horizontally sectioned crown scalp biopsy revealing a scarring alopecia in which the follicular units are entirely replaced with fibrosis and sebaceous glands are absent.

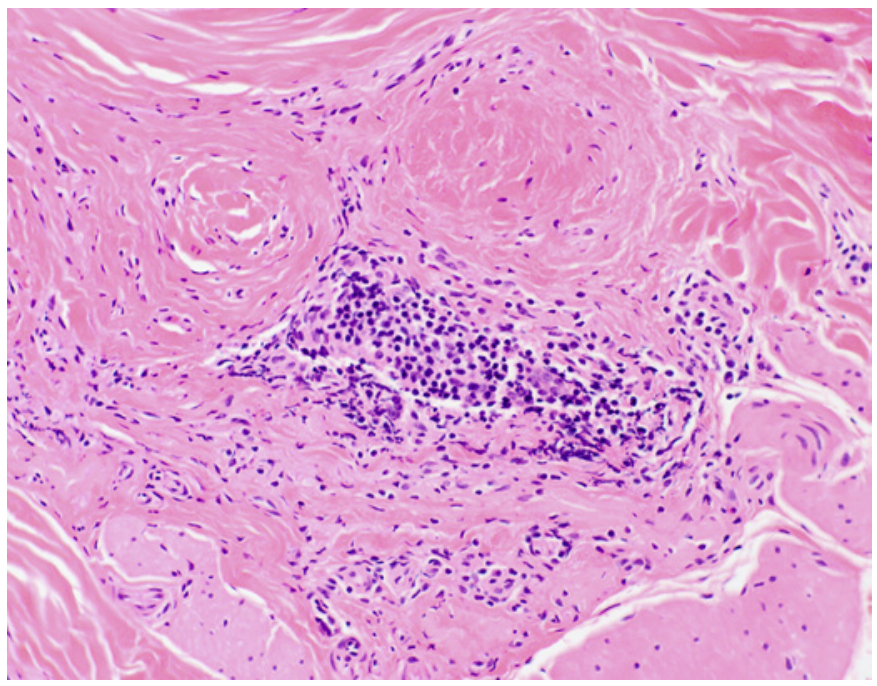

Fig. 4. H\&E stain. High-power image. Inflammation is present, and predominantly consists of lymphocytes with scattered plasma cells.

exposure to halogenated aromatic compounds and ordered PCB (polychlorinated biphenyl) laboratory testing. After numerous attempts to contact his employers, they were unwilling to provide any written documentation of potential chemicals.

At the 6-month follow-up (1 year after the exposure), he displayed rapid expansion of the scarring alopecia process, involving almost one-third of his scalp (Fig. 5). Due to insurance complications, there was a several-month delay in acquiring the PCB laboratory results, and the toxicologists were subsequently unable to prop- erly analyze these results. Over the course of the next 2 years, the patient continued treatment with $200 \mathrm{mg}$ hydroxychloroquine twice daily, intralesional triamcinolone every $2-3$ months, and 1 session of fractional resurfacing ablative laser therapy. Due to his Gorlin syndrome, no other immunosuppressive medications were started.

At the patient's most recent visit ( 3 years after the exposure), more than $95 \%$ of his scalp was involved, with complete loss of follicular ostia (Fig. 6). Just recently, an official policy report detailing chemical exposures at the construction site was released, indicating that the ground-intrusive work resulted in high-concentration exposures to TCE and PCE.

\section{Discussion}

This case represents the first published report on the acute and rapid development of LPP-like alopecia following occupational exposure to TCE/PCE. Classified by the International Agency for Research on Cancer (IARC) as "probably carcinogenic to humans," TCE and PCE have been found in association with a number of known malignancies and other health concerns, though no reports on hair loss in humans have previously been identified [6]. PCE, also known as perchloroethylene or tetrachloroethylene, represents the principal solvent used in dry cleaners in the USA and has been found in association with bladder, kidney, and lymphatic/hematopoietic malignancies in addition to heart disease [6,7]. In mice, only males exposed to drinking water with high concentrations of chlorinated alkanes (including both PCE and TCE) developed tail alopecia and deformation [8]. In comparison to PCE, TCE is primarily used in metal degreasing and has been identified in association with kidney and liver cancer, non-Hodgkin lymphoma, cardiac toxicity, systemic sclerosis, and an increased risk of autoimmune disease [9]. Chronic exposure to TCE in autoimmune-prone $\mathrm{MRL}^{+/+}$mice resulted in a dose-dependent alopecia with dermal inflammation [10].

Although causation cannot be proven, the close temporal relationship and the rapid progression of cicatricial alopecia in an atypical patient demographic support a strong correlation between acute chemical exposure and hair loss. Moreover, a number of medications and chemicals have previously been documented to induce lichen planus-like or lichenoid eruptions via contact exposure, best evidenced by occupational exposure to color film developing agents [11]. As a follicular variant of lichen planus, it is therefore plausible that LPP may also develop in response to chemical exposures, such as TCE and PCE in this case [1].

In addition to exposure to TCE/PCE, our patient's history of Gorlin syndrome may also have contributed, at least in part, to this unique presentation of scarring hair 


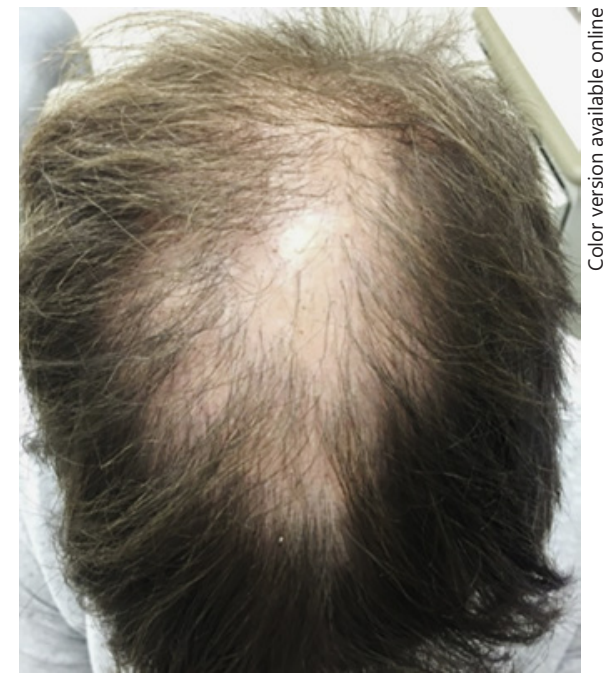

Fig. 5. Scalp at the 6-month follow-up. The crown and vertex scalp demonstrate expanding involvement of scarring alopecia.

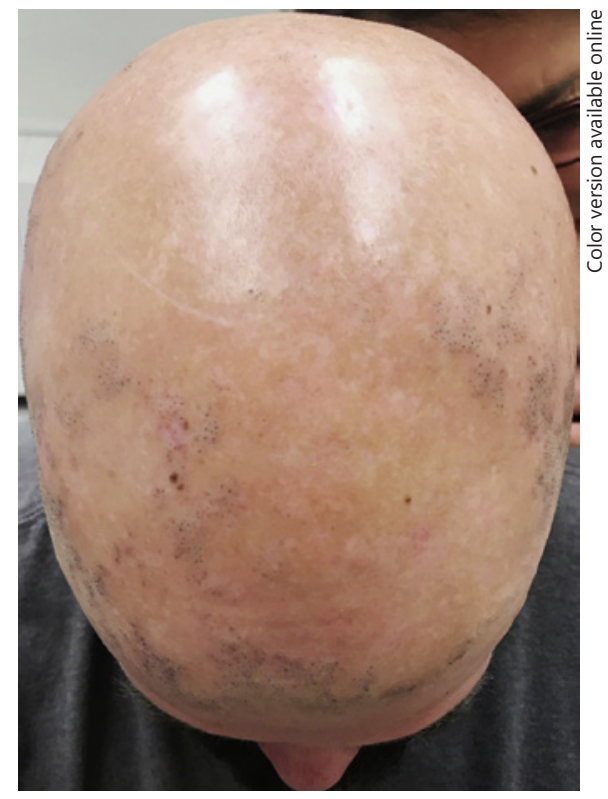

Fig. 6. Scalp at the 28-month follow-up. Rapid expansion of scarring alopecia with complete loss of follicular ostia and postinflammatory pigment alteration, involving $>95 \%$ of the scalp.

loss. Also known as nevoid basal cell carcinoma syndrome, Gorlin syndrome most commonly involves a loss-of-function mutation in the patched $(\mathrm{PTCH} 1)$ gene, resulting in constitutive signaling activity of the Hedgehog $(\mathrm{HH})$ signaling pathway [12]. The HH signaling pathway, furthermore, has been demonstrated to influence hair follicle development and follicle bulge stem cell maintenance [13].

Along the proximal-distal axis of developing hair follicles, PTCH1 and PTCH2 establish an HH signaling gradient during follicular neogenesis and throughout late-stage anagen. This gradient remains critical in regulating an appropriate dose-dependent response for hair follicle development [14]. Furthermore, inactivation of both PTCH1 and PTCH2 during epidermal development leads to complete loss of interfollicular and hair follicle specification and differentiation [15]. Although scarring alopecia is not classically associated with Gorlin syndrome, the genetic predisposition in the context of occupational exposure to TCE/PCE may have contributed to this atypical presentation of cicatricial alopecia. While future studies are necessary to establish causation, clinicians may consider inquiring about occupational exposures in nonclassic patients presenting with scarring hair loss.

\section{Statement of Ethics}

This study complies with the guidelines for human studies and was conducted ethically in accordance with the World Medical Association Declaration of Helsinki. The subject has given his consent to publish details and photos of the case.

\section{Disclosure Statement}

The authors have no conflicts of interest to declare.

\section{Author Contributions}

D.H.M. and M.M.S.: substantial contributions to the conception or design of the work; or the acquisition, analysis, or interpretation of data for the work; all authors: drafting the work or revising it critically for important intellectual content, and final approval of the version to be published; M.M.S.: agreement to be accountable for all aspects of the work in ensuring that questions related to the accuracy or integrity of any part of the work are appropriately investigated and resolved.

\section{References}

Skin Appendage Disord 2019;5:374-378 DOI: $10.1159 / 000501173$
1 Rácz E, Gho C, Moorman PW, Noordhoek Hegt V, Neumann HA. Treatment of frontal fibrosing alopecia and lichen planopilaris: a systematic review. J Eur Acad Dermatol Venereol. 2013 Dec;27(12):1461-70.

2 MacDonald A, Clark C, Holmes S. Frontal fibrosing alopecia: a review of 60 cases. J Am Acad Dermatol. 2012 Nov;67(5):955-61.

3 Kossard S. Postmenopausal frontal fibrosing alopecia. Scarring alopecia in a pattern distribution. Arch Dermatol. 1994 Jun;130(6):7704 . 
4 Tziotzios C, Petridis C, Dand N, Ainali C, Saklatvala JR, Pullabhatla V, et al. Genomewide association study in frontal fibrosing alopecia identifies four susceptibility loci including HLA-B*07:02. Nat Commun. 2019 Mar;10(1):1150.

5 Aldoori N, Dobson K, Holden CR, McDonagh AJ, Harries M, Messenger AG. Frontal fibrosing alopecia: possible association with leave-on facial skin care products and sunscreens; a questionnaire study. Br J Dermatol. 2016 Oct;175(4):762-7.

6 Dry Cleaning, Some Chlorinated Solvents and Other Industrial Chemicals [Internet]. International Agency for Research on Cancer; 1995. Available from: https://www-ncbi-nlmnih-gov.ezp-prod1.hul.harvard.edu/books/ NBK464353/.
7 Callahan CL, Stewart PA, Blair A, Purdue MP. Extended Mortality Follow-Up of a Cohort of Dry Cleaners. Epidemiology. 2019 Mar;30(2): 285-90.

8 Wang FI, Kuo ML, Shun CT, Ma YC, Wang JD, Ueng TH. Chronic toxicity of a mixture of chlorinated alkanes and alkenes in ICR mice. J Toxicol Environ Health A. 2002 Feb;65(3-4): 279-91.

9 Chiu WA, Jinot J, Scott CS, Makris SL, Cooper GS, Dzubow RC, et al. Human health effects of trichloroethylene: key findings and scientific issues. Environ Health Perspect. 2013 Mar; 121(3):303-11.

10 Blossom SJ, Doss JC, Gilbert KM. Chronic exposure to a trichloroethylene metabolite in autoimmune-prone $\mathrm{MRL}+/+$ mice promotes immune modulation and alopecia. Toxicol Sci. 2007 Feb;95(2):401-11.

11 Halevy S, Shai A. Lichenoid drug eruptions. J Am Acad Dermatol. 1993 Aug;29(2 Pt 1): 249-55.
12 Bresler SC, Padwa BL, Granter SR. Nevoid Basal Cell Carcinoma Syndrome (Gorlin Syndrome). Head Neck Pathol. 2016 Jun;10(2): $119-24$.

13 Abe Y, Tanaka N. Roles of the Hedgehog Signaling Pathway in Epidermal and Hair Follicle Development, Homeostasis, and Cancer. J Dev Biol. 2017 Nov;5(4):E12.

14 Adolphe C, Junker JP, Lyubimova A, van Oudenaarden A, Wainwright B. Patched Receptors Sense, Interpret, and Establish an Epidermal Hedgehog Signaling Gradient. J Invest Dermatol. 2017 Jan;137(1):179-86.

15 Adolphe C, Nieuwenhuis E, Villani R, Li ZJ, Kaur P, Hui CC, et al. Patched 1 and Patched 2 redundancy has a key role in regulating epidermal differentiation. J Invest Dermatol. 2014 Jul;134(7):1981-90. 